\title{
Brain Natriuretic Peptide as a Predictor of Major Adverse Cardiac Events after Successful Percutaneous Coronary Intervention
}

\author{
Khaled Ahmed Emam El-Khashab ${ }^{(1)}$ Eman Mahmoud Abd El-Fattah Mohamed ${ }^{(2)}$ Haytham $^{(4)}$ \\ Soliman Ghareeb ${ }^{(3)}$ Mina Samir Kamel ${ }^{(4)}$
}

(1) Professor of cardiovascular medicine, Cardiovascular department, Faculty of medicine, Fayoum University.

(2) Lecturer of cardiovascular medicine, Cardiovascular department, Faculty of medicine, Fayoum University.

(3) Lecturer of cardiovascular medicine, Cardiovascular department, Faculty of medicine, Fayoum University.

(4) Cardiovascular department, 6 October Insurance Hospital and cardiovascular department, Faculty of Medicine, Fayoum university.

Corresponding Author : Prof. Dr. Khaled Ahmed Emam El-Khashab

E-mail address: $\underline{K}$ khashab@gmail.com

Tel: $+\mathbf{2 0 1 0 0 5 1 2 3 6 6 2}$

\section{Abstract}

B-type natriuretic peptide (BNP) has diagnostic and prognostic value in a wide variety of cardiac disorders including heart failure and coronary artery disease; however, it is value in Percutaneous Coronary Intervention (PCI) is not well established.

The aim of the work is to assess whether serum BNP level just before PCI has a predictor value of Major Adverse Cardiac Events (MACE) during hospitalization (as recurrent chest pain, new or worsening heart failure, significant arrhythmia and in-hospital mortality) and after 3 months follow up (as echocardiography assessing left ventricular dysfunction, rest chest pain, hospitalization for Acute Coronary Syndrome (ACS) or heart failure, revascularization and cardiac mortality).

In 82 consecutive patients with Coronary Artery Disease (CAD), plasma BNP levels were measured immediately before successful PCI. Patients were followed for 3 months for the occurrences of MACE. The patients were divided into 2 groups according to occurrence of composite end points of MACE at follow-up; MACE (-) Group: 45 patients who did not have MACE and MACE (+) Group: 37 patients who had MACE.

A statistically significant positive correlation between higher BNP levels and occurrence of MACE (P-value < 0.001)

\section{Keywords}

B-type natriuretic peptide, Coronary artery disease and Percutaneous Coronary Intervention.

\section{Introduction}

The diagnostic value of BNP and NT-proBNP was primarily been investigated in patients with 
heart failure. In a large number of studies, it has consistently been found that BNP and NTproBNP levels are elevated in patients with heart failure; moreover, the levels of BNP and NTproBNP have been found to be related to disease severity, as indicated by functional class (New York Heart Association class), left ventricular systolic ejection fraction and left ventricular diastolic function [1].

Independent of their diagnostic value, several large-scale studies have convincingly demonstrated that high BNP and NT-proBNP levels provide strong prognostic information for an unfavorable outcome (e.g. death, cardiovascular death, and readmission to hospital or cardiac events) in patients with heart failure or asymptomatic left ventricular dysfunction [2].

In multivariable models, BNP and NT-proBNP has shown to be superior to other prognostic parameters and, in some studies, even to be the only independent prognostic factors. Head-tohead studies comparing the diagnostic performance of BNP and NT-proBNP testing were been performed in patients with heart failure and patients with asymptomatic left ventricular dysfunction. These studies showed that both markers performed equally well, with almost identical areas under the receiver operating characteristics curves [3].

Thus, it was concluded from these studies that there is no meaningful difference between the markers in terms of risk stratification in clinical routine.

Originally, BNP and NT-proBNP were considered to be biomarkers of heart failure. More recently, however, there is a growing body of data on the relevance of both markers in CAD. It is widely believed that the underlying pathophysiological process for an increase in BNP and NT-proBNP levels is left ventricular systolic or diastolic dysfunction due to myocardial ischemia leading to increased wall stress. Nevertheless, data derived from experimental studies suggest that there is a direct release of BNP and NT-proBNP from cardiomyocytes in response to myocardial ischemia, independent of ventricular wall stress $[4,5]$.

In agreement with these experimental findings, it has been shown that BNP levels increase even after temporary myocardial ischemia induced by balloon inflation during coronary interventions [6].

The initial studies of BNP in acute coronary syndromes (ACS) were small case-control studies, limited mostly to patients with STelevation MI, who are likely to have at least minor LV dysfunction. More recently, the prognostic application of BNP has been extended to include patients with unstable angina and nonST-elevation myocardial infarction (NSTEMI) [7]. Several studies have come up showing that basal BNP levels in acute MI patients predict short and long term prognosis [8-13].

\section{Patients and methods}

Aim of work is to assess if serum BNP level just before PCI has a predictor value of Major Adverse Cardiac Events (MACE); after successful coronary intervention during hospitalization (as recurrent chest pain, new or worsening heart failure, significant arrhythmia and in-hospital mortality) and after 3 months follow up (as changes in assessment of left ventricular (LV) functions by trans-thoracic echocardiography, rest chest pain, hospitalization for ACS or heart failure, revascularization and cardiac mortality).

This study enrolled 82 consecutive patients admitted to coronary care unit at Cardiovascular Department - Fayoum University Hospital and 6 October Insurance Hospital - Al Doky - Giza, who underwent successful elective or primary percutaneous coronary intervention for Stable CAD (SCAD) or ACS (Unstable Angina, 
NSTEMI and STEMI) in the period from June 2018 to December 2018.

All patients were subjected to detailed history taking, clinical examination, 12-lead ECG, laboratory measurement of BNP within one hour before PCI and serum creatinine level, trans-

\section{Results}

1. This study revealed that there was statistical significance prediction of MACE to BNP level [P-value $<0.001$ ] and diastolic dysfunction during the 3 months follow up [P-value $<0.008$ ].

\begin{tabular}{|c|c|c|c|c|c|c|}
\hline Variables & B & S.E & Wald & Sig. & OR & CI \\
\hline DD & -2.4 & 1.7 & 1.9 & 0.2 & 0.09 & $0.003-2.63$ \\
\hline DD (3m) & 3.8 & 1.4 & 7.1 & $\underline{\mathbf{0 . 0 0 8}}$ & 44 & $2.7-708.2$ \\
\hline $\mathbf{B N P}$ & 4.6 & 0.9 & 26.2 & $\underline{\underline{\mathbf{0 . 0 0 1}}}$ & 103 & $14.5-605.4$ \\
\hline
\end{tabular}

2. There is statistically significant difference in BNP level between degree of diastolic dysfunction with high mean BNP level among grade II diastolic dysfunction at presentation and after 3 months follow up [P-value < 0.05].

\begin{tabular}{|c|c|c|c|c|c|}
\hline \multirow{2}{*}{ Variables } & \multicolumn{2}{c|}{ BNP level } & \multirow{2}{*}{ p-value } & \multirow{2}{*}{ Sig. } \\
\cline { 3 - 4 } & $\begin{array}{c}\text { Mean } \\
\text { (pg/ml) }\end{array}$ & SD & & \\
\hline $\begin{array}{c}\text { Base line } \\
\text { DD }\end{array}$ & Grade I & 27.3 & 10.1 & \multirow{2}{*}{$\underline{\mathbf{0 . 0 0 3}}$} & $\underline{\mathbf{H S}}$ \\
\cline { 2 - 4 } & Grade II & $\underline{\mathbf{4 0 . 8}}$ & 16.8 & & $\underline{\mathbf{H S}}$ \\
\hline $\begin{array}{c}\text { DD after 3 } \\
\text { months }\end{array}$ & Grade I & 25.9 & 9.2 & $\underline{\mathbf{0 . 0 0 1}}$ & $\underline{ }$ \\
\cline { 2 - 4 } & Grade II & $\underline{\mathbf{4 0 . 7}}$ & 12.7 & & \\
\hline
\end{tabular}




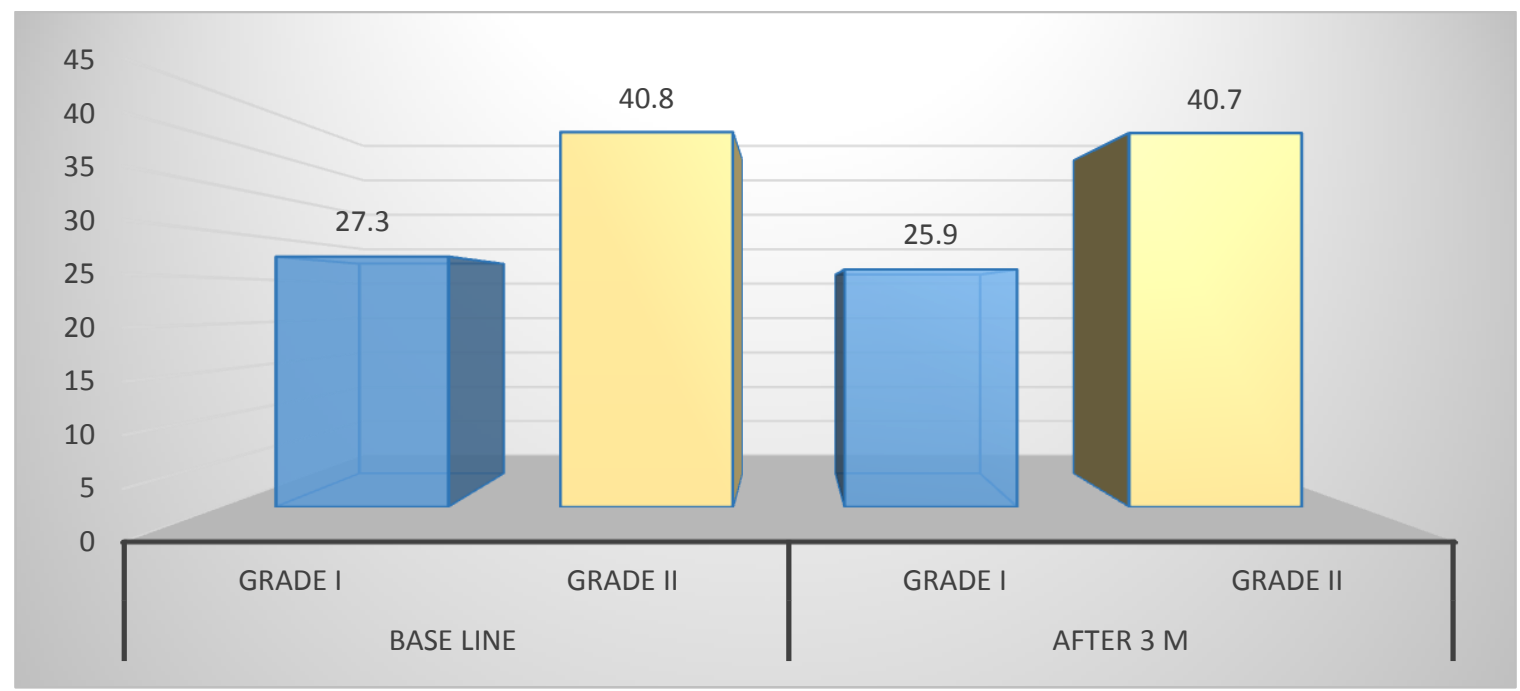

3. There is no statistically significant correlation between BNP level and EF at base line presentation [P-value > 0.2]. On the other hand there is statistically significant negative correlation between BNP level and EF after 3 months; which indicated that increase in BNP level will be associated with decrease in $\mathrm{EF}[\mathrm{P}-\mathrm{value}<0.01]$.

\begin{tabular}{|c|c|c|c|}
\hline \multirow{2}{*}{ EF\% } & \multicolumn{3}{|c|}{ BNP level } \\
\cline { 2 - 4 } & r & p-value & Sig. \\
\hline Base line & -0.15 & 0.2 & NS \\
\hline After 3 months & $\underline{\mathbf{0 . 2 8}}$ & $\underline{\mathbf{0 . 0 1}} *$ & $\underline{\mathbf{S}}$ \\
\hline
\end{tabular}

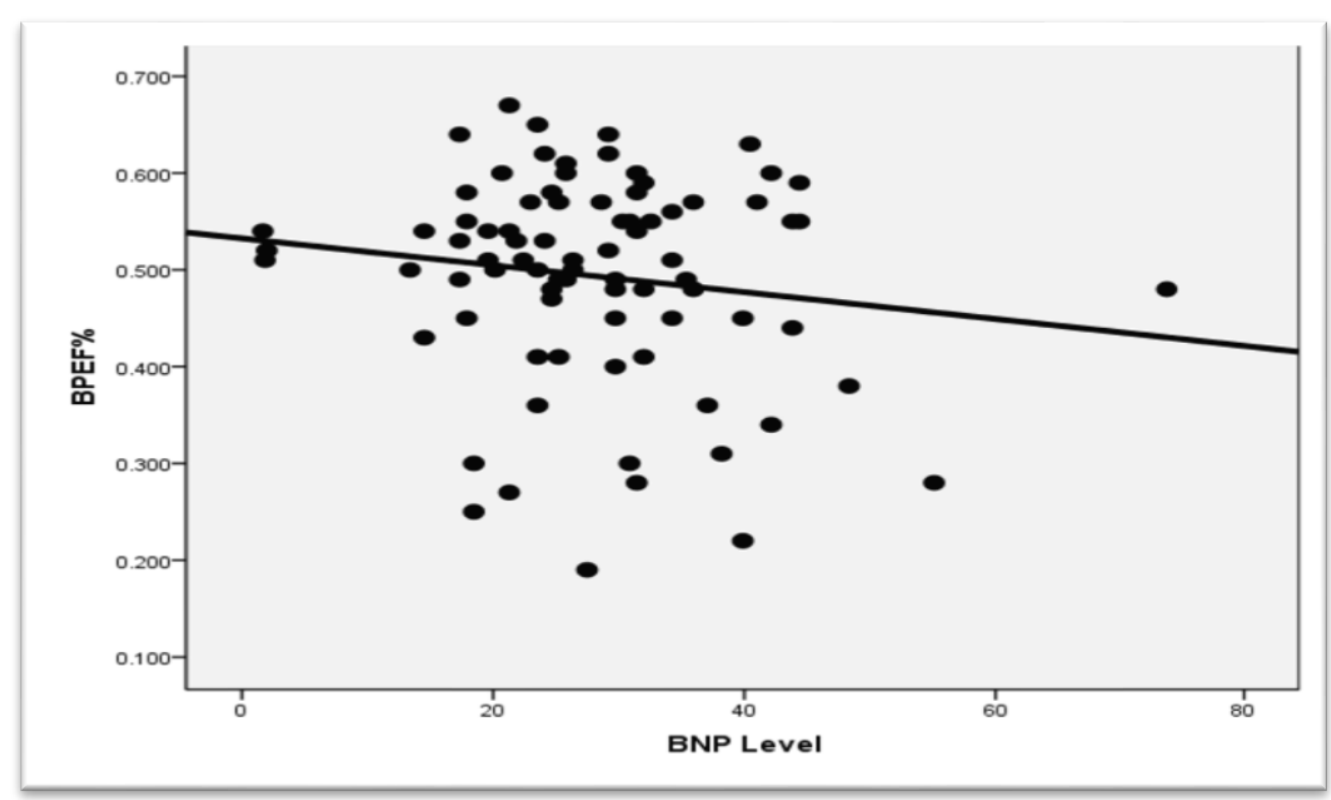




\section{Discussion}

In our study, there was a statistically significant positive correlation between higher BNP levels and occurrence of MACE (P-value < 0.001). 39 patients had higher BNP level for their age; $87.2 \%$ of them had MACE and $91.9 \%$ of the MACE positive group had high BNP level for their age. Plasma BNP levels in MACE Positive group were significantly higher than that of MACE Negative group $[37.02 \mathrm{pg} / \mathrm{ml} \pm 6.5 \mathrm{SD}$ vs. 21.04 $\mathrm{pg} / \mathrm{ml} \pm 10.2 \mathrm{SD}$ and P-value $<0.001]$.

Multiple logistic regression analysis identified that BNP as an independent predictor of MACE during the three months follow up period [OR 103, CI 14.5 - 605.4, P-value <0.001]; considering a BNP level of $37 \mathrm{pg} / \mathrm{ml} \pm 6.5$ is a threshold for patients who are at higher risk for incidence of MACE.

These findings were consistent with the following studies:

Assessment of BNP before PCI and its association with major adverse cardiac events in acute coronary syndrome with heart failure [15]: This study enrolled 150 patients who were followed up for 30 days and concluded that pre PCI BNP and elderly age are strong predictors of major adverse cardiac events at 30 days in patients presenting with acute coronary syndrome with clinically evident heart failure.

Evaluation of B-Type Natriuretic Peptide for Risk Assessment in Unstable Angina/Non-STElevation Myocardial Infarction [16]: This study enrolled 1676 patients who were followed up for six months and concluded that elevated BNP
(>80 pg/ml) at presentation identifies patients with non-ST-elevation ACS who are at higher risk of death and $\mathrm{CHF}$ and adds incremental information to cTnI.

Value of peri-procedural B-type natriuretic peptide levels in predicting cardiac events after elective percutaneous coronary intervention [17]; a study done in 2008 that enrolled 95 patients with SCAD who underwent elective PCI and followed up for 12 months. Blood samples for BNP were obtained before, 1 hour and 24 hours after PCI. The study showed that all measured plasma BNP levels were significantly higher in patients with MACE compared to those free of MACE.

In our study there was no statistically significant correlation between BNP level and Ejection Fraction (EF) at base line presentation [P-value $>0.2]$. On the other hand there is statistically significant negative correlation between BNP level and $\mathrm{EF}$ after 3 months; which indicated that increase in BNP level will be associated with decrease in EF [P-value < 0.01], and there was statistically significant difference in BNP level between degree of diastolic dysfunction with high mean BNP level among grade II diastolic dysfunction at presentation and after 3 months follow up [P-value < $0.05]$.

These findings were consistent with the work done in 2016 in a study [18]; named, Correlation between brain natriuretic peptide levels and the prognosis of patients with left ventricular diastolic dysfunction; that enrolled 708 patients who were followed up for 20-51 months. Endpoints were defined as mortality or 
readmission due to cardiovascular disease, or mortality due to any other reason. The study showed that the prognoses of patients with elevated BNP levels were correspondingly worse

\section{Study Limitations}

1. The small sample size of patients that was enrolled in the study.

2. Our study was not a randomized and controlled study. As a result, we could not exclude the influence of many other unmeasured variables.

3. The dosage of and adherence to medications could not be assessed through our registry. Variations in the use of the appropriate medications could have led to substantial differences in MACE among our patients.

\section{Conclusion}

1. High levels of plasma BNP collected just before successful Percutaneous Coronary Intervention in patients with Coronary Artery Disease are associated significantly with Major Adverse Cardiac Events (MACE) during hospitalization and 3 months follow up.

2. The plasma BNP collected just before successful Percutaneous Coronary Intervention in patients with Coronary Artery Disease is an independent predictor of MACE during the three months follow up period.

\section{Acknowledgement}

First and foremost, I thank Allah who had granted me the ability to accomplish this work. when compared with patients with lower BNP levels. This correlation was demonstrated to be significant in patients with LV diastolic dysfunction.

I would like to express my profound gratitude and deep thanks to PROF. DR. KHALID AHMED EMAM EL-KHASHAB, Professor of Cardiology, Faculty of Medicine, Fayoum University, for his encouragement and valuable instructions.

I would like to give thanks to DR. EMAN MAHMOUD ABD EL-FATTAH MOHAMED, Lecturer of cardiology, Faculty of Medicine, Fayoum University, for her great help and support.

I would like to give thanks to DR. HAYTHAM SOLIMAN GHAREEB, Lecturer of cardiology, Faculty of Medicine, Fayoum University, for his generous supervision, valuable advice, and constant support throughout the whole work. I would like to give thanks to Dr. Mohamed Mansour Abbas Eid, Lecturer of Clinical and Chemical Pathology - Blood Bank Manager Clinical Pathology Department, Faculty of Medicine, Fayoum University, for his great support and efforts.

Words can never express my sincere appreciation to my family for their encouragement and unlimited support.

\section{References}

[1] De Lemos JA, McGuire DK, Drazner MH. Btype natriuretic peptide in cardiovascular disease. Lancet 2003; 362:316-22.

[2] Anand IS, Fisher LD, Chiang YT, et al. Changes in brain natriuretic peptide and norepinephrine over time and mortality and 
morbidity in the Valsartan Heart Failure Trial (Val-HeFT). Circulation 2003; 107:1278-83.

[3] Mueller T, Gegenhuber A, et al. Diagnostic accuracy of $\mathrm{B}$ type natriuretic peptide and amino terminal proBNP in the emergency diagnosis of heart failure. Heart 2005; 91:606-12.

[4] Goetze JP, Christoffersen C, Perko M, et al. Increased cardiac BNP expression associated with myocardial ischemia. FASEB J 2003; 17:1105-7.

[5] Goetze JP, Gore A., et al. Acute myocardial hypoxia increases BNP gene expression. FASEB J 2004; 18:1928-30.

[6] Tateishi J, Masutani M, et al. Transient increase in plasma brain (B-type) natriuretic peptide after percutaneous transluminal coronary angioplasty. Clin Cardiol 2000; 23:776-80.

[7] Omland T, de Lemos JA, Morrow DA, et al. Prognostic value of N-terminal pro-atrial and probrain natriuretic peptide in patients with acute coronary syndromes. Am J Cardiol. 2002; 89: 463-465

[8] De Winter RJ, Stroobants A, Koch KT et.al. Plasma N-terminal pro-B-type natriuretic peptide for prediction of death or nonfatal myocardial infarction following percutaneous coronary intervention.Am J Cardiol. 2004 Dec 15; 94(12):1481-5.

[9] He B, Ding S, Pu J et.al Prognostic value of plasma brain natriuretic peptide and Creactiveprotein in patients with acute coronary syndromes underwent percutaneous coronary intervention ZhonghuaXinXue Guan Bing ZaZhi. 2006 Apr; 34(4):349-52.

[10] Katayama T, Nakashima H, Yonekura T et.al Clinical significance of acute-phase brain natriuretic peptide in acute myocardial infarction treated with direct coronary angioplasty. J Cardiol. 2003 Nov; 42(5):195-200.

[11] Yildirir A, Acikel S, Ertan C et.al Value of peri-procedural B-type natriuretic peptide levels in predicting cardiac events after elective percutaneous coronary intervention. Acta Cardiol. 2008 Feb; 63(1):47-52.

[12] Hong SN, Ahn Y, Hwang SH et.al Usefulness of preprocedural N-terminal pro-brain natriuretic peptide in predicting angiographic noreflow phenomenon during stent implantation in patients with ST-segment elevation acute myocardial infarction.Am J Cardiol. 2007 Aug 15; 100(4):631-4.

[13] Saleh N, Braunschweig F, Jensen J, etal.Usefulness of preprocedural serum $\mathrm{N}$ terminal pro-brain natriuretic peptide levels to predict long-term outcome after percutaneous coronary intervention in patients with normal troponin T levels. Am J Cardiol. 2006 Mar 15; 97(6):830-4.

[14] Measuring left ventricular volume and ejection fraction with the biplane Simpson's method. Heart. 2002; 88(6):559-60.

[15] Abhishek B G, et al., Assessment of BNP before PCI and its association with major adverse cardiac events in ACS with heart failure. Kerala Heart J 2015; 5 (2):24-27.

[16] Morrow, David A. et al. "Evaluation of Btype natriuretic peptide for risk assessment in unstable angina/non-ST-elevation myocardial infarction: B-type natriuretic peptide and prognosis in TACTICS-TIMI 18." Journal of the American College of Cardiology 418 (2003): 1264-72.

[17] Yildirir A et al, Value of peri-procedural Btype natriuretic peptide levels in predicting cardiac events after elective percutaneous coronary intervention. Acta Cardiol. 2008 Feb; 63(1):47-52.

[18] Gong, Hui et al. "Correlation between brain natriuretic peptide levels and the prognosis of patients with left ventricular diastolic dysfunction" Experimental and therapeutic medicine vol. 11, 6 (2016): 2583-2589. 
ISSN: 2536-9482 (Online) Fayoum University Medical Journal El-Khashab et al., 2020,6(1), 28-35 